\title{
Novel breast cancer detection system combining both thermoacoustic (TA) and photoacoustic (PA) tomography using carbon nanotubes (CNTs) as a dual contrast agent
}

Manojit Pramanik, Geng Ku, Changhui Li, Magdalena Swierczewska, Danielle Green, et al.

Manojit Pramanik, Geng Ku, Changhui Li, Magdalena Swierczewska, Danielle Green, Balaji Sitharaman, Lihong V. Wang, "Novel breast cancer detection system combining both thermoacoustic (TA) and photoacoustic (PA) tomography using carbon nanotubes (CNTs) as a dual contrast agent," Proc. SPIE 7177, Photons Plus Ultrasound: Imaging and Sensing 2009, $71772 \mathrm{G}$ (24 February 2009); doi: 10.1117/12.809230

SPIE. Event: SPIE BiOS, 2009, San Jose, California, United States 


\title{
Novel breast cancer detection system combining both thermoacoustic (TA) and photoacoustic (PA) tomography using carbon nanotubes (CNTs) as a dual contrast agent
}

\author{
Manojit Pramanik ${ }^{1}$, Geng Ku ${ }^{1}$, Changhui $\mathrm{Li}^{1}$, Magdalena Swierczewska ${ }^{2}$, Danielle Green ${ }^{2}$, Balaji \\ Sitharaman ${ }^{2 *}$, and Lihong V. Wang ${ }^{1 \dagger}$ \\ 'Optical Imaging Laboratory, Department of Biomedical Engineering, Washington University in St. \\ Louis, St. Louis, MO, USA 63130; \\ ${ }^{2}$ Department of Biomedical Engineering, State University of New York at Stony Brook, Stony \\ Brook, New York, USA 11794
}

\begin{abstract}
We report here a novel breast cancer scanner using microwave and light excitation and ultrasound detection. This combined thermoacoustic and photoacoustic tomography scanner is a nonionizing low cost system that can potentially provide high-resolution, dual contrast (microwave and light absorption) three dimensional images of the breast. Front breast compression will be used in this scanner to alleviate patient discomfort, experienced in side breast compression during traditional X-ray mammography. This scanner will use dry instead of gel ultrasonic coupling. We have also developed a carbon nanotube-based contrast agent for both thermoacoustic and photoacoustic imaging. In the future, targeted molecular photoacoustic and thermoacoustic imaging should be possible using this contrast agent.
\end{abstract}

Keywords: breast cancer, thermoacoustic tomography, photoacoustic tomography, carbon nanotube, single-walled carbon nanotube

\section{INTRODUCTION}

Breast cancer is one of the most common diagnosed cancers among women, accounting for one out of every three cancer diagnoses. In 2005, breast cancer caused 502,000 deaths worldwide (7\% of cancer deaths; almost $1 \%$ of all deaths). In 2008, there were an estimated 182,460 new cases of breast cancer among females, and 1,990 new cases among males in the USA. An estimated 40,480 females died of breast cancer in the USA, as did 450 males. One in 8 women $(12.5 \%)$ has a chance of developing invasive breast cancer and 1 in 35 (3\%) has a chance of breast cancer causing death. To survive breast cancer, early diagnosis and treatment is necessary. ${ }^{1}$

Breast tissue properties such as hardness, optical absorption, and radio-frequency (rf) absorption change during the early stage of breast cancer. Therefore, diagnosis of these subtle changes in the very early stage is essential. Currently, X-ray mammography is the only mass screening tool available, however there are many problems with mamography. ${ }^{2}$ First, it uses ionizing radiation (X-ray) and therefore, frequent screening is not possible. Repeated X-ray exposure can cause problems even after use of low dosage. Second, imaging dense breast and regions close to the chest wall is always cumbersome in X-ray imaging. And finally, it is difficult to diagnose early stage cancer using mammography as the early stage tumors do not have high X-ray contrast.

Recently, sonographic examination of the breast is used routinely with the advent of high-frequency multi-element linear-array transducers. Ultrasonography is also now used in breast imaging centers for the evaluation of breast masses, and as a tool to guide breast interventions. But due to poor soft tissue contrast, the specificity of ultrasound imaging is low, and the current ultrasound scanners can detect and resolve only large breast lesions (few $\mathrm{mm}$ in size). Moreover,

\footnotetext{
*Email: balaji.sitharaman@stonybrook.edu (Carbon nanotube)

${ }^{\dagger}$ Email: 1hwang@biomed.wsutl.edu (Thermoacoustic/photoacoustic tomography)
} 
ultrasound imaging is highly dependent on the operator variability. ${ }^{3}$ Therefore for early cancer detection, ultrasound imaging at its current stage is not a very good option.

Magnetic Resonance Imaging (MRI) has better tissue characterization compared to other imaging modalities. Contrastenhanced MRI is increasingly used in clinics. ${ }^{4}$ In spite of high sensitivity of breast MRI, the specificity is rather variable. Moreover, MRI is very expensive and sometimes uses contrast agents which are not innocuous.

Thermoacoustic and photoacoustic imaging overcome some of the limitations of other imaging modalities by using nonionizing rf and visible/near infrared (NIR) lasers. The rf contrast is very high between malignant tumor tissue and normal human breast tissue (a factor of 4$)^{5}$ compared to a small percent contrast in the case of X-ray imaging. Extra water and sodium ions in the tumor tissue cause the enhanced dielectric property of the malignant tumor. ${ }^{6}$ This contrast does not change much with age, ${ }^{7}$ suggesting that a large contrast could exist even at earlier stages of tumor development. Large contrast is the key motivation for thermoacoustic tomography (TAT) research. TAT combines the high resolution of pure-ultrasound with the high contrast of pure-rf imaging. ${ }^{8,9}$ Pure ultrasound imaging suffers from poor soft-tissue contrast although the resolution is very high and scalable, ${ }^{10,11}$ while pure-rf imaging has poor spatial resolution but high image contrast. ${ }^{12-15}$ Pure-rf imaging has resolution around $10 \mathrm{~mm}$; whereas ultrasound imaging can give resolution as high as $1 \mathrm{~mm}$ or less. TAT combines ultrasound and $\mathrm{rf}$ imaging and gives both high resolution and high soft-tissue contrast. We have previously reported high resolution $(0.5 \mathrm{~mm})$ deep-tissue imaging using TAT. ${ }^{16}$ For breast cancer monitoring the combined high resolution and high contrast imaging is essential.

In TAT/PAT, a short-pulsed microwave/laser source irradiates the tissue. Absorbed microwave/laser energy causes thermoelastic expansion and radiates thermoacoustic/photoacoustic waves from within the irradiated tissue. The microwave/laser heating must be rapid to produce thermoacoustic waves of sufficiently high frequencies. A wide-band ultrasonic transducer is then employed to acquire the thermoacoustic/photoacoustic waves and images are reconstructed to show the tissue microwave/laser absorption information. We have shown experimentally that the spatial resolution is bandwidth- and diffraction-limited by the photoacoustic waves because ultrasonic scattering is weak compared with optical scattering. ${ }^{17,18}$ Therefore, the PAT technique is a unique noninvasive technology for imaging and vascularization quantification, especially oxygen saturation of breast tumors. The optical absorption contrast due to both oxy- and deoxy-hemoglobin relative to the background can be as high as 10:1 or even 100:1, depending on the optical wavelength. ${ }^{18}$ Therefore, PAT may have a major role in breast cancer research and detection by assessing functional and molecular cancer characteristics.

Motivation for combining TAT and PAT: 1) Reduced image acquisition time, 2) inexpensive, and 3) no need for image registration as acquiring two images in the same setup avoids moving and realigning the patient all over again. Moreover, pure ultrasound imaging can also be incorporated easily with the combined TAT and PAT.

Motivation for developing single-walled carbon nanotubes (SWNTs) as a multimodal contrast agent: The developments of PAT/TAT have also concomitantly lead to the development of contrast agents to improve the contrast during very early stages of cancer and for molecular imaging. ${ }^{19,20}$ SWNTs were explored as contrast agents in these studies since they have excellent intrinsic optical ${ }^{21,22}$ and $\mathrm{rf}^{23}$ absorbing properties and thus, may be suitable as multimodal contrast agents for simultaneous TAT and PAT.

\section{METHODS AND MATERIALS}

\subsection{System Description}

The side breast compression, used in X-ray mammography is not very comfortable for the patient. Moreover one needs to take a mammogram from different angles to interpret the images accurately. This breast scanner was designed to minimize the compression pain. The breast will be compressed from the front (nipple side) to give it a cylindrical shape. This technique has advantages in three ways. First, transducers can scan around the cylindrical breast for a full 360 degrees and along the length to obtain a full three-dimension (3D) data set. Full 3D reconstruction can be done to view the breast in 3D. Second, compression from the front is less painful than one from the side. Third, microwave/laser irradiation from the front of the cylindrically compressed breast can potentially penetrate deep enough to image near the chest wall. 
The breast holder is made of LDPE (low density polyethylene), which has low ultrasound absorption and an acoustic impedance close to that of water ( $\sim 1.5$ at room temperature). LDPE minimizes the loss of ultrasound signal due to a mismatched boundary. The breast is inserted into the front opening of the scanner. A supporting plate made of acrylic pushes the breast from the rear opening of the scanner to obtain a cylindrical shape. After the compression, a brass retaining ring holds the supporting plate in position. The microwave/laser source is kept behind the scanner, and a horn antenna is pushed inside the scanner from the opening to irradiate rf/light on the compressed breast. An aluminum cylinder (rotating cylinder) with many holes holds the ultrasonic transducers around the breast holder cylinder. During data collection, the rotating cylinder is turned by a worm-gear mechanism and a stepper motor. The whole setup is mounted inside a stainless steel frame filled with mineral oil that facilitates the coupling of ultrasound to the transducer and also lubricates the worm gear. Mineral oil is also a non microwave absorbing material and since mineral oil is transparent, the light absorption is also very small. The scanner is placed on a height- and angle- adjustable metal frame. A patient will be positioned, sitting on a chair and leaning onto the scanner.

The microwave/laser assembly is placed on the back side of the scanner behind the support plate. The breast is illuminated by either microwave or laser alternately for TAT/PAT. The microwave is delivered to the breast using a horn antenna, whereas the laser is delivered by a free space optical assembly. Some parts of the laser illumination system are incorporated inside the microwave horn antenna. As a result, mechanical switching between the microwave and laser source is not needed. The switching is electronic and instantaneous - once a TAT image is collected using microwave illumination, the microwave is switched off and the laser is switched on electronically to collect a PAT image. To be more precise, a prism and a ground glass plate are placed inside the microwave horn antenna. Both are non-metallic and therefore will not affect the microwave illumination. A drilled $\sim 10 \mathrm{~mm}$ diameter hole in one narrow wall of the horn antenna delivers the light. The laser beam is broadened by a concave lens placed outside the hole on the horn antenna, then reflected by the prism, and homogenized by the ground glass.

\subsection{Microwave Source}

A $3.0 \mathrm{GHz}$ microwave source produces pulses of width $0.5 \mu$ s with a repetition rate of up to $100 \mathrm{~Hz}$. The breast is illuminated using an air-filled pyramidal horn type antenna (WR284 horn antenna W/EEV flange, HNL Inc.) with an opening of $7.3 \times 10.7 \mathrm{~cm}^{2}$. The pulse energy is estimated to be around $10 \mathrm{~mJ}(=20 \mathrm{~kW} \times 0.5 \mu \mathrm{s})$ (within the IEEE safety standards ${ }^{24}$ ). The horn antenna is designed to transport the $\mathrm{TE}_{10}$ mode of EM waves, so the electric field is parallel (or nearly parallel for a horn) to the surface of either narrow side ( $y$-polarized in our system) and approaches zero near the inner surface of either narrow wall. By contrast, the electric field is nonzero near the surface of either wide wall. Therefore, opening the light delivery hole on the narrower side of the horn antenna (or waveguide) minimizes power leakage.

\subsection{Laser Source}

A Q-switched Nd:YAG laser with a repetition rate of $10 \mathrm{~Hz}$ provides $6.5 \mathrm{~ns}$ wide $(6.5 \mathrm{~ns}$ at $1064 \mathrm{~nm}$ wavelength, $5 \mathrm{~ns}$ at $532 \mathrm{~nm}$ wavelength) laser pulses. The laser system can provide $850 \mathrm{~mJ}$ maximum output energy at $1064 \mathrm{~nm}$ wavelength (400 mJ at $532 \mathrm{~nm}$ wavelength). In the NIR spectral region, absorption of melanin is relatively low and is not expected to limit the delivery of light into the breast even for African American patients, as demonstrated in a previous study of diffuse optical imaging of the breast. ${ }^{25}$ The laser beam is expanded by a concave lens, homogenized by ground glass, and then directed onto breast. This type of beam expansion scheme has been used extensively before. ${ }^{17,26,27}$ The incident laser fluence on the tissue surface is controlled to $<20 \mathrm{~mJ} / \mathrm{cm}^{2}$ to conform to the American National Standards Institute (ANSI) standards. ${ }^{28}$

\subsection{Detection of Ultrasound}

For detecting the ultrasound signal, $13 \mathrm{~mm} / 6 \mathrm{~mm}$ diameter active area non-focused transducers operating at $2.25 \mathrm{MHz}$ central frequency (ISS $2.25 \times 0.5 \mathrm{COM}$, Krautkramer) are used. The transducers are scanned around the sample a full 360 degrees to collect data at different angular positions. The signal is first amplified by a low-noise pulse amplifier (5072PR, OlympusNDT), then filtered electronically, and finally recorded using a digital data acquisition card (14 bit Gage Card). When a microwave is the illumination source, a delay/pulse generator (SRS, DG535) triggers the microwave pulses and synchronizes the data sampling of the Gage Card. On the other hand, during laser illumination the sync out of the laser system synchronizes the laser pulses and the data sampling of the Gage card. A simple delay-andsum algorithm is used to reconstruct TAT/PAT images. 


\subsection{Single-walled carbon nanotubes synthesis}

A diblock copolymer templating method was used to coat Fe coated on Si wafers. ${ }^{29}$ The wafers were placed in a 3 inch quartz reaction chamber (Easy Tube 2000, First Nano) and heated in $\mathrm{Ar}$ to $900^{\circ} \mathrm{C}$. The chamber was filled with $\mathrm{H}_{2}$ for 2 minutes and $\mathrm{CH}_{4}$ was then added to the gas flow as the carbon feedstock for 20 minutes to initiate the growth of SWNTs. Subsequently, the carbon feedstock was switched off and the furnace was cooled to room temperature. Raman spectroscopy (LabRAM Aramis, Horiba JvonYvon) at $633 \mathrm{~nm}$ excitation and transmission electron microscope (TEM) imaging (FEI Tecnai12 BioTwinG ${ }^{2}$ ) at $80 \mathrm{kV}$ were used to characterize the SWNTs. Figures 1(a) and 1(b) show the TEM and the Raman spectra of SWNTs, respectively.
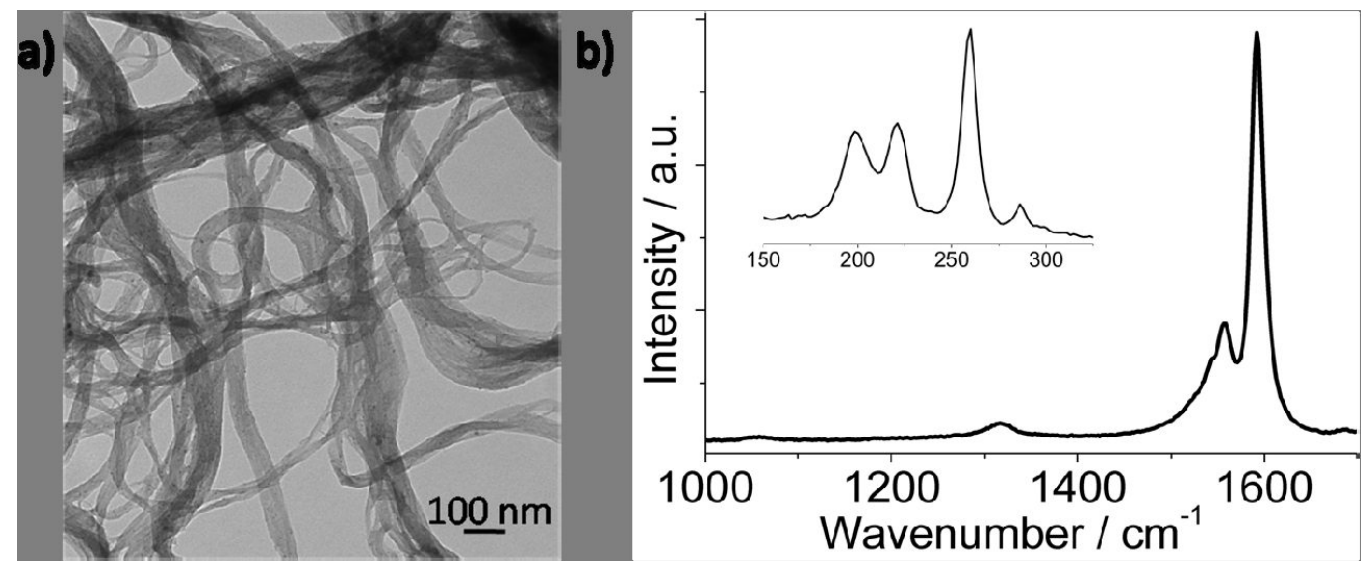

Figure 1. (a) TEM of the SWNTs (b) D-band and G-band Raman spectra of the SWNTs. The inset shows the radial breathing modes.

\section{RESULTS AND DISCUSSIONS}

\subsection{Integrating TAT/PAT}

The imaging quality with and without integrating the optics inside the horn antenna was tested. We observed that there was not much effect of the insertion of optical components inside the horn antenna. Therefore, the placement of optical components (here, the prism and the ground glass plate) inside the horn antenna to deliver light has insignificant effect on the imaging, and as a result we were able to integrate both light and microwave delivery.

\subsection{Dry Coupling}

In conventional ultrasound imaging, coupling-gel is used between the body surface and the ultrasonic transducer, but in our breast scanner dry-coupling will be used. Since the cylindrically compressed breast will be held tightly inside the LDPE breast holder, there will not be a significant air gap to hinder ultrasound propagation. A thin rubber balloon filled with mineral oil was used as a breast model to test the feasibility of dry coupling. The balloon was placed inside the breast holder chamber and then compressed to a cylindrical shape that fits tightly inside the LDPE breast holder cylinder. This experiment was done with the scanner tilted at 45 degrees. There was a small air gap at the top portion of the breast holder cylinder. Even with this small air gap between the balloon and the breast holder cylinder, when we made a transmission ultrasound measurement around the breast holder cylinder we saw very good ultrasound coupling. Figure 2 shows the transmission ultrasound coupling at different depths of the compressed balloon. From figure 2 it can be seen that except near the end of the compressed balloon $(2 \mathrm{e} \& 2 \mathrm{f})$, the remaining planes have good ultrasonic transmission, meaning good ultrasonic coupling. Even for those few planes where we do not have good coupling, we have good coupling over $80 \%$ of the area. Since we do tomography and collect data at different angular positions for reconstruction, losing data over $20 \%$ of the area will not affect the reconstructed image significantly. 

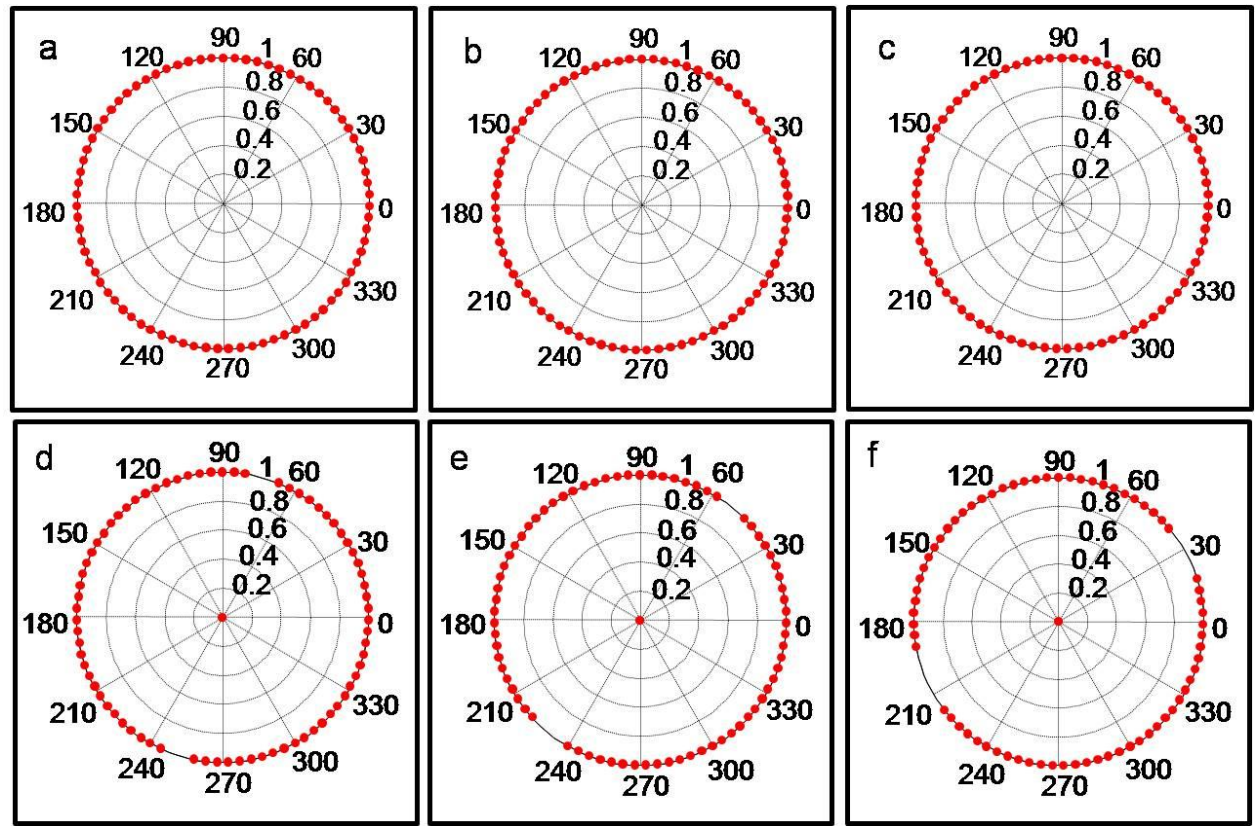

Figure 2. The transmission of ultrasound around the breast holder at different depths with a compressed balloon filled with mineral oil inside the breast holder chamber. The red dots represent the transmission of ultrasound in that region. Depths: (a) $3 \mathrm{~mm}$, (b) $8 \mathrm{~mm}$, (c) $1.0 \mathrm{~cm}$, (d) $1.4 \mathrm{~cm}$, (e) $1.7 \mathrm{~cm}$, (f) $2 \mathrm{~cm}$.

\subsection{Phantom Experiments}

A tissue phantom experiment was also done. Porcine fat was used as the background medium mimicking the fatty breast tissue, and water-based agar gel was used to model the inhomogeneity (target) in the background. The target gel objects were made of $2 \%$ agar, $2 \%$ salt and $96 \%$ water. Salt was added to increase the microwave absorption. A total of five target objects of diameter $\sim 6 \mathrm{~mm}$ were buried inside $\sim 7 \mathrm{~cm}$ diameter porcine fat. Two targets were made of clear gel. The remaining three targets were made of black gel; the color was obtained by mixing black India ink during the preparation of the agar gel. The location of the target objects inside the base fat is shown in figure 3(a), a photograph of the phantom. The sample was placed inside the breast holder chamber, and the chamber was then filled with mineral oil, an ultrasound coupling medium. Figure 3(b) shows the reconstructed PAT image with $6 \mathrm{~mm}$ diameter active area ultrasonic transducers. Figures 3(c) and 3(d) show TAT images with $6 \mathrm{~mm}$ and $13 \mathrm{~mm}$ diameter active area transducers, respectively. As expected, we can clearly see all five objects in the TAT images (Figs. 3c, 3d). TAT is based on microwave absorption and all five objects are made of water based gel that is much more opaque to microwave than the background fat tissue. In contrast, PAT images reveal only three black target objects (Fig. 3b) as the contrast in PAT imaging is dependent on light absorption coefficient. Thus the combination of both PAT and TAT will provide more information about the target objects.

\subsection{Characterizing SWNTs}

An initial assessment was made for a variety of carbon nanostructures (fullerenes, single-walled carbon nanotubes, multiwalled carbon nanotubes and graphite microparticles) with different inner and outer diameters as well as on graphite microparticles. LDPE tubes filled with each type of samples were placed in the scanner and TA/PA signals were recorded. Only SWNTs showed a significant increase in TA signal compared to deionized (DI) water, and a significant increase in PA signal compared to rat blood. The increase in TA and PA signals were shown by pristine SWNTs (mass of Fe is $\sim 20 \%$ of total weight of SWNT sample) as well as purified SWNTs (mass of Fe is $\sim 0.5-1 \%$ of the total weight of SWNT sample), clearly indicating that the observed effects were due to the SWNTs and not due to the presence of iron. Since only SWNTs showed a significant difference in both PA and TA signals, they were used for the phantom imaging experiments. 

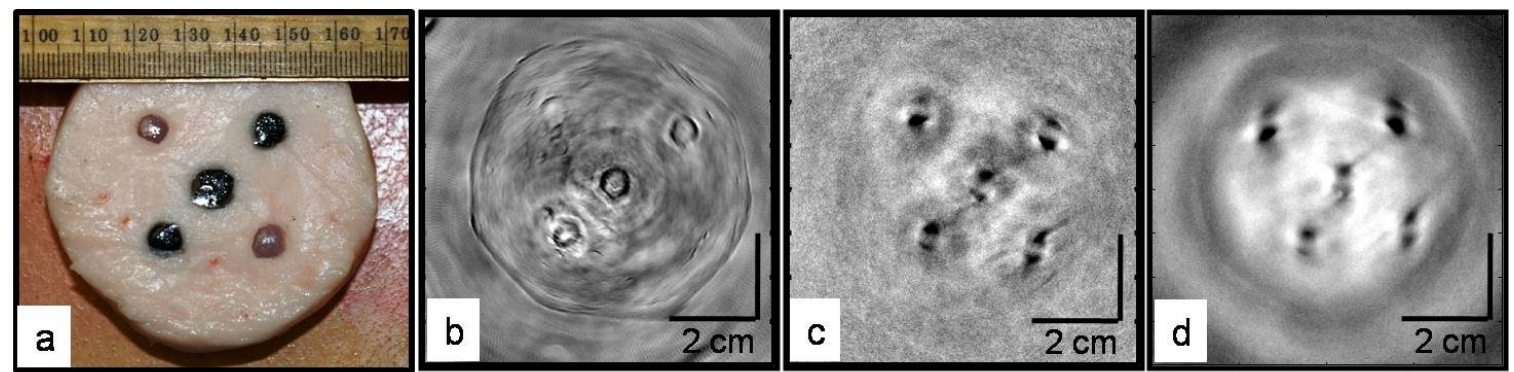

Figure 3. Cross-sectional TAT and PAT images of a tissue mimicking phantom obtained from the breast scanner. Five water-based agar gel targets were embedded inside a porcine fat base. (a) Photograph of the phantom, the smallest tick is $1 \mathrm{~mm}$. Two targets were clear objects and the remaining three targets were black. (b) PAT image obtained at 1064 $\mathrm{nm}$ wavelength using a $6 \mathrm{~mm}$ diameter active area transducer. (c) TAT image obtained using a $6 \mathrm{~mm}$ diameter active area transducer. (d) TAT image obtained using a $13 \mathrm{~mm}$ diameter active area transducer.

\subsection{SWNTs as TA contrast agent}

Figure 4 shows the TA signals from an LDPE tube filled with various samples. As we can see, the peak-to-peak TA signal amplitudes generated from the tube filled with mineral oil is insignificant (at the noise level) compared to the signal from DI water, therefore we conclude that there is no acoustic signal generated from the LDPE tube (mineral oil does not absorb microwave therefore it will not produce any TA signal) as expected. So now onwards all the signals we see will be assumed to be generated from the sample placed inside the tube. The peak-to-peak TA signal amplitude generated from the $1 \%$ Pluronic ${ }^{\circledR}$ F127 surfactant (1 gm of Pluronic ${ }^{\circledR}$ in 100 gm of water) is also similar to that of DI water confirming that the surfactant does not generate any additional TA signal. The peak-to-peak TA signal amplitudes generated from the DI water and $1 \mathrm{mg} / \mathrm{ml} \mathrm{SWNTs}$ are $73 \mathrm{mV}$ and $276 \mathrm{mV}$, respectively. The variation of TA signals with the concentration of SWNTs is also seen in the figure. The peak-to-peak TA signal amplitudes from different concentrations of SWNTs solution is as follows: $0.1 \mathrm{mg} / \mathrm{ml}=65 \mathrm{mV}, 0.25 \mathrm{mg} / \mathrm{ml}=103 \mathrm{mV}, 0.5 \mathrm{mg} / \mathrm{ml}=301 \mathrm{mV}, 0.75$ $\mathrm{mg} / \mathrm{ml}=263 \mathrm{mV}, 1 \mathrm{mg} / \mathrm{ml}=276 \mathrm{mV}$. A TA signal of more than four times strong is observed from SWNTs compared to that of DI water. Since water is a known microwave absorber in the human body and produces TA signal, observing a stronger TA signal from SWNTs than DI water confirms that SWNTs can work as contrast agents for TA imaging.

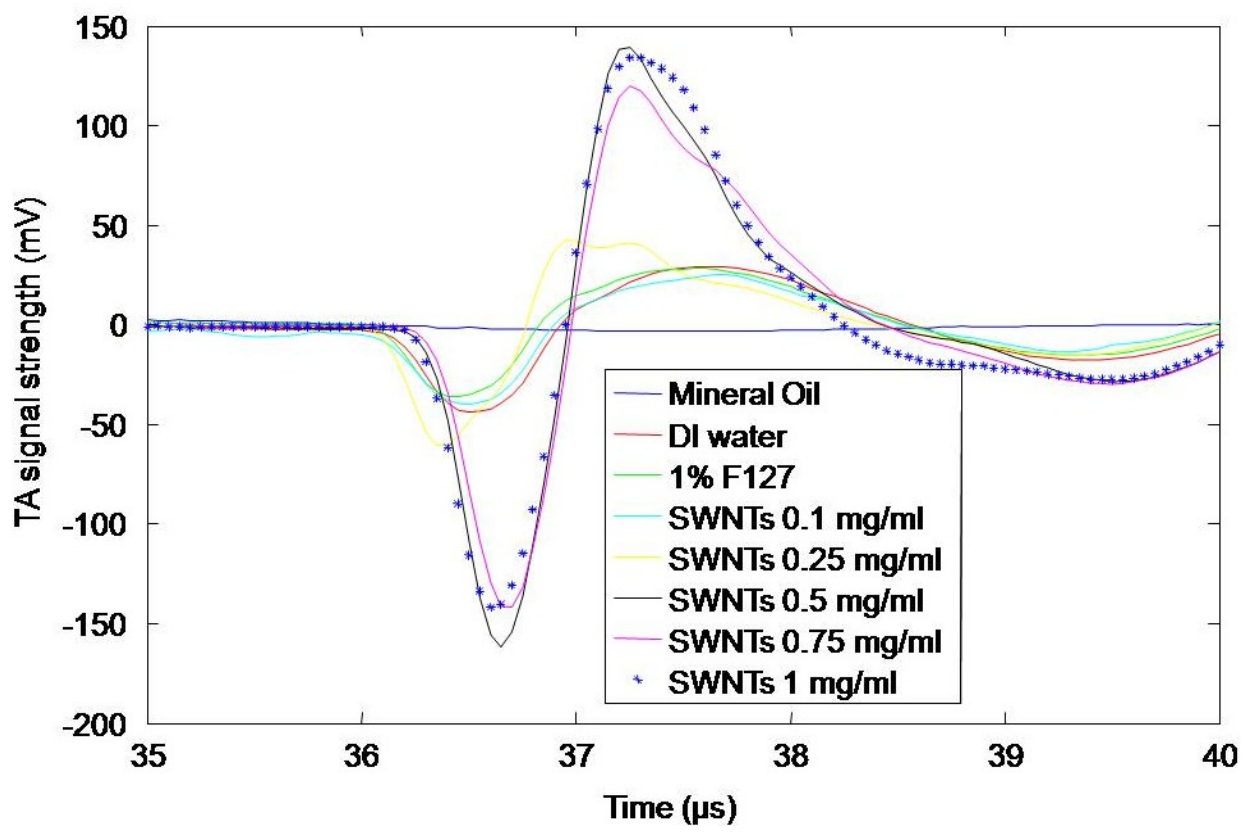

Figure 4. TA signal from LDPE tube filled with different samples. 


\subsection{SWNTs as PA contrast agent}

Figure 5 shows the PA signal generated from the LDPE tube filled with different samples. A $532 \mathrm{~nm}$ wavelength light source was used. The peak-to-peak PA signal amplitudes generated by blood and $1 \mathrm{mg} / \mathrm{ml} \mathrm{SWNTs}$ are $46 \mathrm{mV}$ and 145 $\mathrm{mV}$, respectively. The variation of PA signals with the concentration of SWNTs is also seen in the figure. The peak-topeak TA signal amplitudes from different concentration SWNTs solution are as follows: $0.1 \mathrm{mg} / \mathrm{ml}=86 \mathrm{mV}, 0.25$ $\mathrm{mg} / \mathrm{ml}=124 \mathrm{mV}, 0.5 \mathrm{mg} / \mathrm{ml}=107 \mathrm{mV}, 0.75 \mathrm{mg} / \mathrm{ml}=107 \mathrm{mV}, 1 \mathrm{mg} / \mathrm{ml}=145 \mathrm{mV}$. More than a three time PA signal strength difference is observed from SWNTs compared to that of rat blood. Since blood is a known light absorber in the human body and produces a PA signal, observing a PA signal from SWNTs stronger than blood confirms that SWNTs can work as a contrast agent for PA imaging.

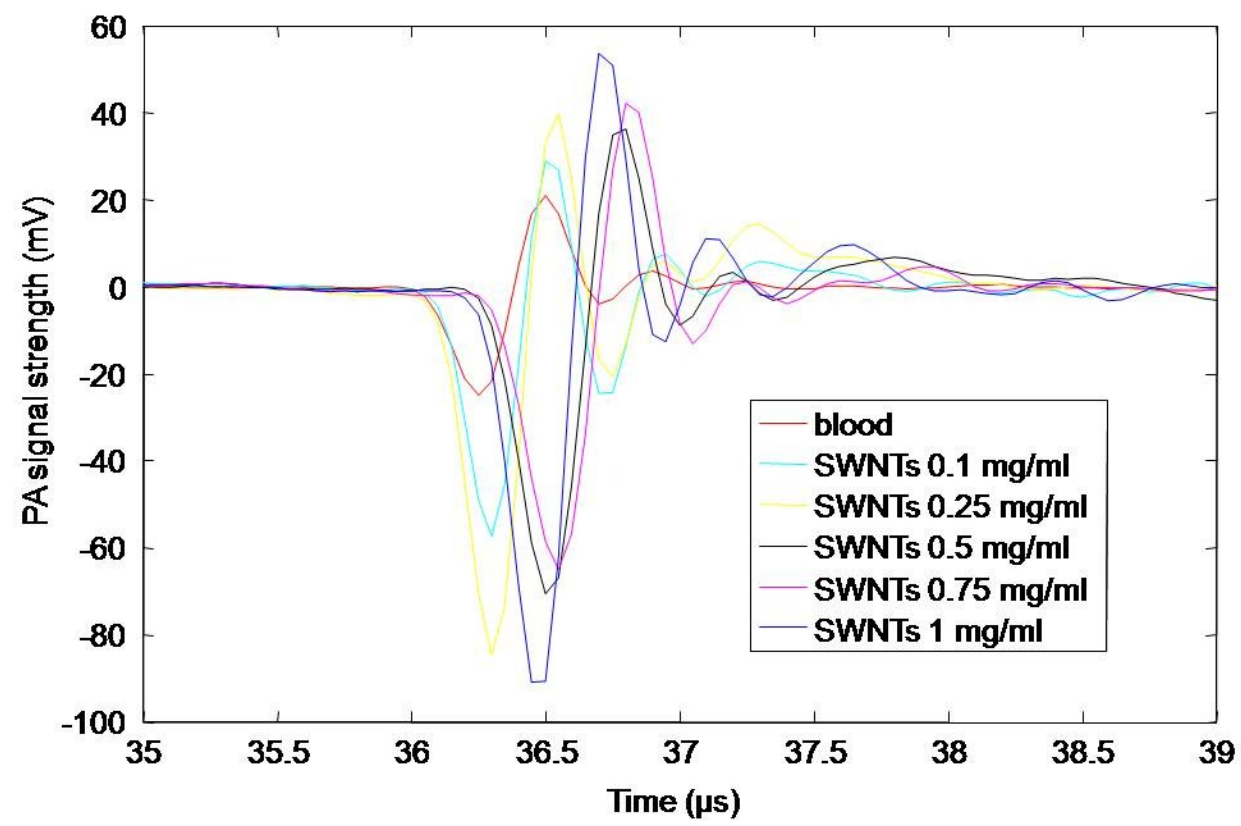

Figure 5. PA signal from LDPE tubes filled with different samples.

\section{SUMMARY}

We have integrated two imaging modalities, TAT and PAT, and showed tissue phantom imaging with it. The combined system can provide additional functional information for the diagnosis of breast cancer. This system is more comfortable for the patient as it uses front compression instead of side compression and dry coupling instead of gel coupling. The low imaging contrast at the early stage of cancer will be compensated by using a contrast agent. SWNTs will work as a contrast agent for both modalities. In the future, targeted molecular thermoacoustic and photoacoustic imaging will be possible using this system and the contrast agent.

\section{ACKNOWLEDGEMENTS}

This work was supported by National Institutes of Health grants R01 EB000712 and R01 NS046214 (LVW) and the Office of the Vice President of Research at Stony Brook University (SB). The authors would like to thank Dr. Oleg Gang and Dr. Huming Xiong at the Center for Functional Nanomaterials, Brookhaven National Laboratory for access to the AFM, Mr. Tom Salagaj and Mr. Christopher Jensen at FirstNano/CVD Equipment Corporation for access to their CVD facilities and Dr. Eunah Lee at Horiba JvonYvon, Edison, NJ for the Raman Spectroscopy measurements. 


\section{REFERENCES}

[1] Harris, J. R., Lippman, M. E., Veronesi, U., and Willett, W., "Medical Progress .2. Breast-Cancer," New England Journal of Medicine, 327, 390-398 (1992).

[2] Nass, S. J., Henderson, I. C., and Lashof, J. C., [Mammography and Beyond: Developing Techniques for the Early Detection of Breast Cancer], Nat. Acad. Press, Washington, D.C. (2001).

[3] Lehman, C. D., Isaacs, C., Schnall, M. D., Pisano, E. D., Ascher, S. M., Weatherall, P. T., Bluemke, D. A., Bowen, D. J., Marcom, P. K., Armstrong, D. K., Domchek, S. M., Tomlinson, G., Skates, S. J., and Gatsonis, C., "Cancer yield of mammography, MR, and US in high-risk women: Prospective multi-institution breast cancer screening study," Radiology, 244, 381-388 (2007).

[4] Kuhl, C., "The current status of breast MR imaging - Part I. Choice of technique, image interpretation, diagnostic accuracy, and transfer to clinical practice," Radiology, 244, 356-378 (2007).

[5] Homburger, F., The physiopathology of cancer, 2d ed. [New York]: Hoeber-Harper, 1959.

[6] Kruger, R. A., Miller, K. D., Reynolds, H. E., Kiser, W. L., Reinecke, D. R., and Kruger, G. A., "Breast cancer in vivo: Contrast enhancement with thermoacoustic CT at $434 \mathrm{MHz}$ - Feasibility study," Radiology, 216, 279-283 (2000).

[7] Kremkau, F. W., [Diagnostic ultrasound: principles and instruments], 4 ed., W.B. Saunders, Philadelphia (1993).

[8] Steen, E., and Olstad, B., "Volume rendering of 3-D medical ultrasound data using direct feature mapping," IEEE Transactions on Medical Imaging, 13, 517-525 (1994).

[9] Lin, J. C., "Frequency optimization for microwave imaging of biological tissues," Proc. IEEE, 73, 374-375 (1985).

${ }^{[10]}$ Larsen, L. E., Jacobi, J. H., and IEEE Microwave Theory and Techniques Society., Medical applications of microwave imaging. New York: IEEE Press, 1986.

[11] Fear, E. C. and Stuchly, M. A., "Microwave system for breast tumor detection," IEEE Microwave and Guided Wave Letters, 9, 470-472 (1999).

[12] Hawley, M. S., Broquetas, A., Jofre, L., Bolomey, J. C., and Gaboriaud, G., "Microwave Imaging of Tissue Blood Content Changes," Journal of Biomedical Engineering, 13, 197-202 (1991).

[13] Meaney, P. M., Paulsen, K. D., and Chang, J. T., "Near-field microwave imaging of biologically-based materials using a monopole transceiver system," IEEE Trans. Microwave Theory Tech., 46, 31-45 (1998).

[14] Hagness, S. C., Taflove, A., and Bridges, J. E., "Three-dimensional FDTD analysis of a pulsed microwave confocal system for breast cancer detection: Design of an antenna-array element," IEEE Transactions on Antennas and Propagation, 47, 783-791 (1999).

[15] Caorsi, S., Frattoni, A., Gragnani, G. L., Nortino, E., and Pastorino, M., "Numerical Algorithm for DielectricPermittivity Microwave Imaging of Inhomogeneous Biological Bodies," Medical \& Biological Engineering \& Computing, 29, Ns37-Ns44 (1991).

[16] Jacobi, J. H., and Larsen, L. E., "Microwave time delay spectroscopy imagery of isolated canine kidney," Medical Physics, 7, 1-7 (1980).

[17] Ku, G., Fornage, B. D., Jin, X., Xu, M. H., Hunt, K. K., and Wang, L. V., "Thermoacoustic and photoacoustic tomography of thick biological tissues toward breast imaging," Technology in Cancer Research \& Treatment, 4, 559-565 (2005).

[18] Wang, L. H. V., and Wu, H.-i, [Biomedical Optics: Principles and Imaging], Wiley, (2007).

[19] Ottobrini, L., Ciana, P., Biserni, A., Lucignani, G., and Maggi, A., "Molecular imaging: A new way to study molecular processes in vivo," Molecular and Cellular Endocrinology, 246, 69-75 (2006).

[20] De La Zerda, A., Zavaleta, C., Keren, S., Vaithilingam, S., Bodapati, S., Liu, Z., Levi, J., Smith, B. R., Ma, T. J., Oralkan, O., Cheng, Z., Chen, X. Y., Dai, H. J., Khuri-Yakub, B. T., and Gambhir, S. S., "Carbon nanotubes as photoacoustic molecular imaging agents in living mice," Nature Nanotechnology, 3, 557-562 (2008).

[21] Hughes, M. E., Brandin, E., and Golovchenko, J. A., "Optical absorption of DNA-carbon nanotube structures," Nano Letters, 7, 1191-1194 (2007).

[22] Berciaud, S., Cognet, L., Poulin, P., Weisman, R. B., and Lounis, B., "Absorption spectroscopy of individual singlewalled carbon nanotubes," Nano Letters, 7, 1203-1207 (2007).

[23] Gannon, C. J., Cherukuri, P., Yakobson, B. I., Cognet, L., Kanzius, J. S., Kittrell, C., Weisman, R. B., Pasquali, M., Schmidt, H. K., Smalley, R. E., and Curley, S. A., "Carbon nanotube-enhanced thermal destruction of cancer cells in a noninvasive radiofrequency field," Cancer, 110, 2654-2665 (2007).

[24] IEEE standard for safety levels with respect to human exposure to radio frequency electromagnetic fields $3 \mathrm{kHz}$ to $300 \mathrm{GHz}$, IEEE Std C95.1, 1999 Edition. 
[25] Monsees, B., Destouet, J. M., and Totty, W. G., "Light scanning versus mammography in breast cancer detection," Radiology, 163, 463-465, 1987.

[26] $\mathrm{Ku}, \mathrm{G}$. and Wang, L. H. V., "Deeply penetrating photoacoustic tomography in biological tissues enhanced with an optical contrast agent," Optics Letters, 30, 507-509 (2005).

[27] Wang, Y. W., Xie, X. Y., Wang, X. D., Ku, G., Gill, K. L., O'Neal, D. P., Stoica, G., and Wang, L. V., "Photoacoustic tomography of a nanoshell contrast agent in the in vivo rat brain," Nano Letters, 4, 1689-1692 (2004).

[28] Laser Institute of America, American National Standard for Safe Use of Lasers ANSI Z136.1-2000 (American National Standards Institute, Inc., New York, NY, 2000).

[29] $\mathrm{Fu}, \mathrm{Q} .$, Huang, S. M., and Liu, J., "Chemical vapor depositions of single-walled carbon nanotubes catalyzed by uniform $\mathrm{Fe}_{2} \mathrm{O}_{3}$ nanoclusters synthesized using diblock copolymer micelles," Journal of Physical Chemistry B, 108, 6124-6129 (2004). 